\title{
SOCIO-DEMOGRAPHIC FACTORS AS MODERATORS OF ATTITUDE TOWARDS COPING AMONG CANCER AND CARDIAC PATIENTS
}

\author{
Mohd. Ashik Shahrier*, Md. Enamul Haque**, Md. Azibar Rahman*** \\ *Lecturer, Department of Psychology, University of Chittagong. ${ }^{1}$ \\ ** Professor, Department of Psychology, University of Rajshahi. \\ *** Lecturer, Department of Psychology, Shalua Degree College, Charghat, Rajshahi. \\ Corresponding Author, Cell: 01718-183582, Email: ashikpsy@cu.ac.bd
}

\begin{abstract}
The study was designed to explore the attitude towards coping of cancer and cardiac patients as related to certain socio-demographic factors like gender and SES. Purposively selected 360 respondents constituted the sample of the present study. Attitude towards Coping Scale (Rahman, 2010) was used for the collection of data. The sample was equally divided into three categories (cancer. cardiac, normal) on the basis of type of individuals ( $\mathrm{N}=120$ for each category).Again they were equally subdivided into male and female on the basis of gender ( $\mathrm{N}=60 \mathrm{for}$ each group).Each category was again equally subdivided into lower middle and upper middle on the basis of SES (N=30 for each group). Results analyzed through ANOVA revealed that the main effects for type of individuals, gender and SES were statistically significant. That is, both cancer and cardiac patients expressed lower coping attitudes as compared to normal individuals, females expressed lower coping attitudes as compared to males and lower middle SES individuals expressed lower coping attitudes as compared to upper middle SES. Again the two way interactions between type of individuals and gender, and type of individual and SES were statistically significant.
\end{abstract}

Key Words: Coping, type of individuals, socio economic status, gender, cancer, cardiac.

সারংশ: আর্থ-সামাজিক বিভিন্ন উপাদান যেমন: লিঙ্গ এবং আর্থ-সামাজিক অবস্থার প্রেক্ষিতে ক্যান্সার এবং কার্ডিয়াক আক্রান্ত রোগীদের মোকাবেলাধর্মী মনোভাব যাচাইয়ের জন্য বর্তমান গবেষণাটি করা হয়। উদ্দেশ্যমূলকভাবে নির্বাচিত ৩৬০ জন উত্তরদাতাকে গবেষণার নমুনা হিসাবে নেয়া হয়। উপাত্ত সংগ্রহের জন্য নোকাবেলাধর্মী মনোভাব মাপক (রহমান, ২০১০) ব্যবহৃত হয়। ব্যক্তির ধরন (ক্যান্সার, কার্ডিয়াক, স্বাভাবিক) এর উপর ভিত্তি করে নমুনাকে তিনটি ক্যাটাগরিতে ভাগ করা হয় (প্রতি দলে ১২০ জন)। এরপর তাদেরকে লিঙ্গের ভিত্তিতে দু'ভাগে (ছেলে = ৬০ জন, হেয়ে = ৬০ জন) ভাগ করা হয়। আবার, তাদেরকে আর্থ-সামাজিক অবস্থানুযায়ী নিয্ন মধ্যবিত্ত ও উচ্চ মধ্যবিত্ত (প্রতি দলে ৩০ জন করে) এ দু’ভাগে ভাগ করা হয়। ভেদাংক বিশ্লেষণ দ্বারা প্রাপ্ত ফলাফল থেকে দেখা যায়, ব্যক্তির ধরণ, লিঙ্গ এবং আর্থ-সামাজিক অবস্থার মুখ্য প্রভাব পরিসংখ্যানিকভাবে তাৎপর্যপূর্ণ হয়েছে। ক্যান্সার এবং কার্ডিয়াক উভয় রোগীরাই স্বাভাবিক ব্যক্তিদের তুলনায় কম মোকাবেলাধর্মী মনোভাগ পোষণ করে, মেয়েরা ছেলেদের তুলনায় কম মোকাবেলাধর্মী মনোভাব পোষণ করে, নিম্নমধ্যবিত্তরা উচ্চমধ্যবিত্তদের তুলনায় কম মোকাবেলাধর্মী মনোভাব পোষণ করে। আবার, ব্যক্তির ধরণ ও লিঙ্গ এবং ব্যক্তির ধরণ ও আর্থ-সামাজিক অবস্থার দ্বি-মুখী পারস্পরিক প্রভাবসমূহও পরিসংখ্যানিকভাবে তাৎপর্যপূর্ণ হয়েছে।

\section{Introduction}

Coping is defined as the process of managing external or internal demands that exceed the resources of the person. It is a complex and multidimensional process that is sensitive to both the environment and the personality of the individual. It is the process of trying to manage and master stressful events. Coping with a stressful event is a dynamic process. Successful coping depends on a combination of problem-solving ability and emotional self-regulation (Cohen \& Lazarus, 1973).People coping well solve problems by changing aspect of their environment that is harmful or threatening; they regulate their emotions by maintaining a positive self-image and satisfactory relationships with others. The importance of family and friends to our health stems from the social support they provide in stressful times. This support may be tangible assistance, information, and emotional support when our self-esteem is threatened (Scheier et al. 1989). Kobasa(1984) concluded that the combination of commitment, control, and challenge makes a person "hardy". Such people are able to defuse potentially stressful situations. Techniques for reducing the body's physiological responses to stress include progressive relaxation, autogenic training and biofeedback. People can also learn to manage stress through programs that change their cognitive and behavioural responses. In a representative community study eight distinct coping strategies have emerged. These include: confrontative coping, seeking social support, planned problem-solving, self-control and distancing, positive appraisal, accepting responsibility and escape or avoidance. Researchers judge coping according to its effectiveness in reducing psychological distress. If negative emotions such as anxiety and depression are reduced by a coping effort, the coping effort is judged to be successful (Lazarus \& Folkman, 1984).The ways through which a person copes with a stressful event and whether or not that coping will ultimately be successful in reducing stress depend upon a variety of coping resources. Internal resources consist of coping styles and personality attributes. External resources include money, time, social support, and other life stressor that may be occurring at the same time. All of these factors interact with each other to determine coping processes.

Research indicates that by changing their behaviour, cancer and cardiac patients can greatly reduce their risk. Patients develop a number of control-related beliefs with respect to cancer or coronary heart disease. They may believe, as do many cancer patients, that they can prevent 
a recurrence of the disease through good habits or even merely with power force of will (Taylor et al. 1984). Heart patients may believe that by avoiding stressful situations, they will avoid exacerbating their disorder. A much more adaptive response to a stressful event of chronic illness like cancer or CHD is to engage in acts that will generate positive affect. Research by Stone and his colleagues (1994) indicates that positive events enhance the immune system over a longer time period than a negative event. Feelings of personal control appear to be important in the practice of preventive health behaviour, illness related behaviours and adjustment to medical procedures. Positive cognition also helps to reduce chronic illness like cancer or coronary heart disease. Holland (1977) found that, among patients with advanced stage cancers, emotional support and reassurance were more effective than medication in dealing with the depression, anxiety and mourning that accompany reactions to life threatening illness. People who interact closely with others are better able to avoid stress due to cancer a CHD than those who remain isolated from interpersonal contract (Williams et al. 1991).A review of the available literature corresponding to the attitudes towards coping among cancer and cardiac patients in relation to socio demographic factors like gender and SES has been put forward in this section.

Richardson et al. (1997) conducted a study to differentiate the effects of imagery and support on coping, life attitudes, immune function, quality of life, and emotional well-being after breast cancer. Results indicated that for all women interventions and perceived social support improved coping skills (seeking support), and enhance meaning in life. Support boosted overall coping and death acceptance. When comparing imagery with support, imagery participants tended to have less stress, increased vigor, and improved functional and social quality of life. Carver et al. (1993) using the model of coping found that acceptance and the use of humor predicted lower distress while denial and disengagement predicted greater distress among breast cancer patients/survivors during the first year of the illness experience. Esterling et al. (1996) found evidence that social support may modulate the effect of chronic stress on immune function. Social support may be a key moderator of the effect of psychosocial stress on cancer development. Earlier investigations of coping with cancer focused on documenting the frequency of use of and the role played by psychological defense mechanisms (e.g., projection, suppression, denial, displacement, reaction formation) in adapting to the disease (Gleser et al. 1969; Cooper et al. 1978). Goldzweig et al. (2009) studied the effects of gender difference among middle-aged cancer patients and their healthy spouses. This study assesses psychological distress, coping and social support among middle-aged couples, where one of the partners was diagnosed with colon cancer. In this study men (healthy or sick) were found to be more distressed than their wives $(p<0.0001)$. Men also reported receiving more support from their wives than did the female spouses $(p<0.0005)$.A study by Endler et al. (1999) investigated differences in illness-specific coping strategies, selfefficacy, and perceived control over illnesses in adults (18-72 years) reporting acute ( $n=137 ; 41$ males, 96 females) and chronic ( $n=137 ; 41$ males, 96 females) health problems. Analyses showed that individuals with acute illnesses scored higher on general self-efficacy than individuals with chronic illnesses. People with chronic illnesses were more likely to use a combination of emotional preoccupation, instrumental and distraction coping strategies, whereas people with acute illnesses used palliative coping strategies to a greater extent. Carver et al. (1993) found that acceptance predicted lower levels of distress among women during the first year after treatment for breast cancer. In terms of coping, survivors who used denial as a coping strategy also worried more about cancer. Aldwin et al. (1996) found that the most consistently significant correlates between any of the survivors' characteristics and coping were their age. The older the survivor the less likely they are to use planning, denial, venting or seeking social support to cope with cancer disease.

In case of cardiac disease, French et al. (2001) mentioned that if coronary patients believe the cause of their problem to be genetic or hereditary they may hold a fatalistic attitude and continue harmful behaviours (for example, smoking, poor diet) in contrast to those who believes their illness may have been caused by their poor lifestyle and will be more willing to entertain and persist in efforts to change their lifestyle and are more likely to engage in exercise and dietary changes. Mahler \& Kulik (1998) have examined cardiac patients' sense of control over their illness and found supporting evidence, demonstrating that lower levels of perceived control led to negative health affects and higher levels of acceptance and benefits beliefs have been related to better psychological and physical heath status. Barnoy et al. (2005) conducted a study to examine the influence of correspondence in informational coping style (monitoring vs. blunting) on the psychological reaction of 98 married couples where one is a cancer patient and the other is the main caregiver. The results showed that for female patients, correspondence in monitoring with their spouse was associated with their better psychological reaction, while for male patients correspondence in blunting was associated with their 
better psychological reaction. Harburg et al. (1973) and James and Kleinbaum (1976) found that persons living in high stress and low SES areas experience a greater number of anger-provoking situations and evidence different coping patterns (i.e., a greater tendency toward anger inhibition) than do persons living in low stress and high SES areas. Social stress, psychological distress, and psychosocial support affect the adjustment of lower and upper middle SES breast cancer patients, influence their experience of and adherence to medical treatment, and may effect the course of the disease. A study by Spiegel (1997) indicate that clear and open communication, expression of appropriate emotion, and collaborative planning and problem-solving enhance the adjustment of high SES breast cancer patients. Conversely, influences that isolate lower middle SES breast cancer patients from others or undermine support can have adverse medical and psychological consequences.

\section{Objective of the Study}

The broad objective of the study was to explore the attitudes towards coping of cancer and cardiac patients in Bangladesh as related to certain socio-demographic factors like gender and SES.

\section{Rationale of the study}

A chronic disease like cancer or coronary heart disease has an impact on all aspects of a patient's life. The patient's psychological state is almost certainly affected in that the diagnosis of a chronic illness can produce extreme fear and anxiety or depression. But physicians, psychiatrists or significant people of the society believe that appropriate behaviour toward cancer or cardiac patients requires a cheerful, optimistic front, so the patients would feel better. Actually the person who becomes ill due to cancer or cardiac disease must process the incoming information, attend to whatever physical symptoms are present, and correctly interpret them for coping with the disease effectively. By changing their behaviour towards the disease and to engage in acts that will generate positive affect, cancer and cardiac patients can greatly reduce their risk. To know whether the changes in self-concept, personal relationships, and work that can result from chronic disease will affect the sufferers in a positive or negative manner in coping with the diseases would be the major concern of this study. Cancer or cardiac disease may also be responsible for strained, disrupted interpersonal relationships which have a unique impact on the patient's psychological well-being and adjustment to the illness. Problems with social relationships may occur because of the fear and stigma associated with the illness, as well as the absence of a set of social norms concerning the appropriate way to behave around a cancer or cardiac patient. By considering these important aspects this study would be an attempt to focus on the differential coping attitudes of cancer and cardiac patients due to their self perceptions and society's perceptions and interpretations about their illnesses as related to certain socio demographic factors.

\section{Hypotheses of the Study}

$\mathbf{H}_{1}$ : Both cancer and cardiac patients would express lower coping attitudes as compared to normal individuals.

$\mathbf{H}_{2}$ : Females would express lower coping attitudes as compared to males.

$\mathbf{H}_{3}$ : Lower middle SES individuals would express lower coping attitudes as compared to upper middle SES individuals.

\section{Materials and Methods}

Sample: A total of 360 respondents constituted the sample of the study. At first a sheet was set for collecting the personal information of the participants such as name, age, educational qualification, occupation, monthly income, religion, husband/parental income, marital status, health wellbeing, types of illness etc. To identify the participants as cancer or cardiac individuals, a questionnaire consisting of 10 items named 'Cancer-Cardiac Criteria Questionnaire' developed by the researcher was administered in question form through 'yes' or 'no' approach to the respondents. Through this questionnaire individuals were diagnosed whether they were cancer or cardiac patients or not and other questions of this questionnaire indicated how positively or negatively cancer or cardiac patients took their disease and dealt with it. Individuals who were not suffering any chronic disease or any other illness were diagnosed as normal individuals and it was confirmed through the personal information sheet that ensured whether they were suffering from any chronic illness or not. Through this procedure and after the application of 'Cancer-Cardiac Criteria Questionnaire' some of the subjects were found as cancer patients, some were cardiac and some individuals were found to be normal individuals. Thus, finally three categories of subjects were selected. From them, 120 Ss were purposively selected as cancer patients, 120 cardiac patients and 120 as normal individuals. Then, the subjects were sub-divided into males and females comprising 60 participants for each group. Again, these participants were divided into two groups' i.e. upper middle SES and lower middle SES comprising 30 participants for each group. From the three SES related 
questions of personal information sheet, the SES level of the respondents was determined. These SES related questions consisted of the respondents' education level, occupation and monthly income. Through these three questions, the researcher with his own setup criteria for SES based on respondents' education level, occupation and monthly income by considering the present socioeconomic condition of Bangladesh identified subjects into two categories such as upper-middle and lower middle SES. Thus, the total subjects comprised of 360 respondents selected purposively for this study and after that the main instrument 'Attitude towards Coping Scale' was administered on the sample to know their attitude towards coping.

\section{Instruments}

The following measures were used to collect data in the present study :(1) Personal information sheet (PIS), (2) Cancer-Cardiac Criteria Questionnaire (CCCQ), (3) Attitude towards Coping (ATC) Scale.

Personal Information Sheet (PIS): A questionnaire was set for collecting the personal information of the subjects, such as name, age, educational qualification, occupation, monthly income, religion, husband/parental income, marital status, health wellbeing, types of illness, SES etc.

\section{Attitude towards Coping (ATC) Scale}

In this study Attitude towards Coping (ATC) Scale constructed by Rahman, A. (2010) was used for collection of data. This scale contains 24 items divided into 6 dimensions, four items for each dimension. These dimensions were: coping with illness, coping with stress, coping with medical, coping with stressful events, coping with environment and coping with the financial accommodation. The split half reliability was computed with odd and even numbers of those 24 items scores and the Pearson's ' $r$ ' was found 0.80. To find out the validity of the scale, correlation between the total ATC scores with the scores of each dimension were computed which ranged from 0.41 to 0.77 .

\section{Procedure of Data Collection}

In this study, the investigator utilized two measures i.e. PIS and ATC scale to each of the 120 subjects separately for cancer patients, cardiac patients and normal individuals. The investigation was conducted on a sample of three categories - (i) cancer patients, (ii) cardiac patients and (iii) normal individuals. Data of all patients were collected from different medical college hospitals \& health institutes of Rajshahi and Dhaka cities. Data of cancer patients were collected from Rajshahi Medical College Hospital (18 respondents); Rajshahi Cancer Hospital and Research Centre (5 respondents); Dhaka Ahsania Mission Cancer Hospital (19 respondents); Mudabbir Cancer Care Centre, Dhaka (7 respondents); National Cancer Institute Mohakali, Dhaka (11 respondents); Square Hospital, Dhaka (20 respondents); Appollo Hospital, Dhaka (17 respondents); Central Hospital, Dhaka (13 respondents); Delta Hospital, Dhaka (10 respondents). Data of Cardiac patients were collected from Rajshahi Medical College Hospital (32 respondents), Rajshahi Heart Foundation (16 respondents), Dhaka National Cardiac Hospital (36 respondents), Dhaka Ibrahim Cardiac Hospital \& Research Institute (36 respondents); data of normal individuals were collected from different parts of Rajshahi (60 respondents) and Dhaka (60 respondents) cities. The participants took half an hour to fill up ATC questionnaire. However, the participants were very much eager to fill up the questionnaire. Though they were always busy for something but they co-operated the investigator to fill up the scale. The investigator was able to make a rapport with the participants. They were told that these collections of data would help them coping with stress unless and until they expressed their views and opinions frankly. Thus, they spontaneously responded to all the questions.

\section{Scoring Procedure}

The data of the present study were collected through ATC scale. This scale contains 24 items divided into 6 dimensions, four items for each dimension. There are five alternative responses to each item. These are: (i) strongly agree, (ii) agree (iii) neutral, (iv) disagree and (v) strongly disagree. The responses to various positive items were scored in such a way that 5, 4, 3, 2 \& 1 was respectively given for the five above mentioned alternatives. The responses to various negative items were scored in such a way that $1,2,3,4$ \& 5 was respectively given for the five above mentioned alternatives. Then the total score of each respondent is obtained by adding the scores of all 24 items. Thus for 24 items, the score ranged from 24 to 120 . Thus the highest score indicated highest coping attitudes and the lowest score indicated lowest stress attitudes. Hence, the midpoint was

$=\frac{\text { Highest score }+ \text { Lowest score }}{2}=\frac{120+24}{2}=72$

The scores above this mid point were indicative of higher coping attitudes and the scores under this mid point were indicative of lower coping attitudes. 


\section{Design of the Study}

This is a study of persons with the disease (cancer and cardiac) of interest and a suitable control group of persons without the disease i.e. normal individuals (comparison group, reference group). The potential relationship of a suspected risk factor to the disease i.e. attitude towards stress is examined by comparing the diseased (cancer or cardiac) and non diseased (normal) subjects with regard to how frequently the factor is present in each of the groups (diseased and non diseased) based on certain demographics like gender and SES. Thus, case control design was used for the present study.

\section{Results}

The study used three independent factors such as type of individuals, gender and SES. Type of individuals was divided into cancer patient, cardiac patient and normal individual. Gender was divided into males and females. SES included upper middle SES and lower middle SES. These independent factors were indicative to show differential attitude towards stress. The data of the present study were analyzed through Analysis of Variance (ANOVA).

Table 1 Showing summary of factorial ANOVA involving Type of Individual, Gender and SES on the scores of Attitude towards Coping Scale.

\begin{tabular}{l|l|l|l|l|}
\hline \multicolumn{1}{c}{ Source of Variance } & \multicolumn{1}{c|}{ Sum of Squares } & df & \multicolumn{1}{c|}{ Mean Square } & F \\
\hline Type of Individual (A) & 11484.29 & 2 & 5742.14 & $35.00^{* *}$ \\
Gender (B) & 9486.40 & 1 & 9486.40 & $57.82^{* *}$ \\
SES (C) & 2711.51 & 1 & 2711.51 & $16.53^{* *}$ \\
AB & 2119.40 & 2 & 1059.70 & $6.46^{* *}$ \\
AC & 1262.02 & 2 & 631.01 & $3.85^{*}$ \\
BC & 10.00 & 1 & 10.00 & 0.06 \\
ABC & 160.07 & 2 & 80.03 & 0.49 \\
Within Cell (Error) & 57099.47 & 348 & 164.08 & \\
Total & 84333.16 & 359 & & \\
\hline
\end{tabular}

$\mathrm{P}<0.05^{*}, \quad \mathrm{P}<0.01^{* *}$

The results reported in table- 1 showed that main effects for type of individual $(F=35.00, d f=1 / 348, p<0.01)$, gender $(\mathrm{F}=57.82, \mathrm{df}=1 / 348, \mathrm{p}<0.01)$ and $\mathrm{SES}(\mathrm{F}=16.53, \mathrm{df}=1 / 348, \mathrm{p}<0.01)$ were statistically significant. It was also found that interaction effects of a two-way analysis of variance involving type of individual \& gender $(\mathrm{F}=6.46, \mathrm{df}=1 / 348$, $\mathrm{p}<0.01)$ and type of individual and SES $(\mathrm{F}=3.85, \mathrm{df}=1 / 348, \mathrm{p}<0.05)$ were also statistically significant.

Table 2 Showing overall mean scores and significant mean differences for the main effect of type of individual on the scores of Attitude towards Coping Scale ( $\mathrm{N}=120$ for each group).

\begin{tabular}{|c|c|c|c|c|c|c|c|c|}
\hline \multirow{2}{*}{ Comparison groups } & \multirow{2}{*}{$\begin{array}{c}\text { Mean } \\
\text { Scores }\end{array}$} & Cancer & Cardiac & Normal & \multirow{2}{*}{$\mathrm{r}$} & \multirow{2}{*}{$\frac{q .95}{q .99}$} & \multirow{2}{*}{$\sqrt{m s / n}$} & \multirow{2}{*}{$\begin{array}{c}\text { Critical } \\
\text { value }\end{array}$} \\
\hline & & 54.30 & 64.45 & 67.52 & & & & \\
\hline Cancer & 54.30 & & $10.15^{*}$ & $13.22 *$ & 3 & $\frac{3.31}{4.12}$ & 1.59 & $\frac{5.26}{6.55}$ \\
\hline Cardiac & 64.45 & & & 3.07 & 2 & $\frac{2.77}{3.64}$ & 1.59 & $\frac{4.40}{5.78}$ \\
\hline Normal & 67.52 & & & & & & & \\
\hline
\end{tabular}

Note: Mean differences were computed using Newman-Keuls formula, (Winer, 1977, PP. 191-195). $\mathrm{P}^{* *}<0.05 ; \mathrm{P} *<0.01$. - $\mathrm{r}=$ Number of Steps between ordered scores

- The critical values of q.95 and q.99 has been obtained from the distribution of Studentized Range Statistics Prepared by Leon Harter; Donald S. Clemm and Eugene H. Guthrie and reported in Winer, 1971. An inspection of mean scores reported in table-2 showed that regardless of gender and SES, cancer patients $(\mathrm{M}=54.30)$ expressed significantly lower coping attitude as compared to cardiac patients $(\mathrm{M}=64.45)$. Similarly, cancer patients $(\mathrm{M}=54.30)$ expressed significantly lower coping attitude as

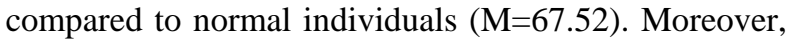
cardiac patients $(\mathrm{M}=64.45)$ expressed significantly lower coping attitude as compared to normal individuals ( $\mathrm{M}=67.52)$. 
Table 3 Showing overall mean scores and significant mean difference for the main effect of gender on the scores of Attitude towards Coping Scale ( $\mathrm{N}=180$ for each group).

\begin{tabular}{l|c|}
\hline \multicolumn{1}{|c|}{ Gender } & Mean Scores \\
\hline Male & 67.22 \\
Female & 56.96 \\
\hline
\end{tabular}

An inspection of mean scores reported in table-3 showed that regardless of type of individual and SES, females $(M=56.96)$ expressed significantly lower coping attitude as compared to males $(\mathrm{M}=67.22)$.
Table 4 Showing overall mean scores and significant mean difference for the main effect of SES on the scores of Attitude towards Coping Scale ( $\mathrm{N}=180$ for each group).

\begin{tabular}{|c|c|}
\hline SES & Mean Scores \\
\hline Upper Middle SES & 64.83 \\
Lower Middle SES & 59.35 \\
\hline
\end{tabular}

An inspection of mean scores reported in table-4 showed that regardless of type of individual and gender, lower middle SES individuals ( $M=59.35)$ expressed significantly lower coping attitude as compared to the upper middle SES individuals ( $\mathrm{M}=64.83)$.

Table 5 Showing overall cell means and their significant mean differences of two-way interaction involving type of individual and gender on the scores of Attitude towards Coping Scale.

\begin{tabular}{|c|c|c|c|c|c|c|c|c|c|c|c|}
\hline \multirow[t]{2}{*}{$\begin{array}{c}\text { Comparison } \\
\text { groups }\end{array}$} & \multirow[t]{2}{*}{$\begin{array}{l}\text { Mean } \\
\text { Scores }\end{array}$} & $\begin{array}{l}\text { Cancer } \\
\text { Male }\end{array}$ & $\begin{array}{l}\text { Cancer } \\
\text { Female }\end{array}$ & $\begin{array}{l}\text { Cardiac } \\
\text { Male }\end{array}$ & $\begin{array}{l}\text { Cardiac } \\
\text { Female }\end{array}$ & $\begin{array}{l}\text { Normal } \\
\text { Male }\end{array}$ & $\begin{array}{l}\text { Normal } \\
\text { Female }\end{array}$ & \multirow{2}{*}{$\mathrm{r}$} & \multirow[t]{2}{*}{$\frac{q .95}{q .99}$} & \multirow[t]{2}{*}{$\sqrt{m s / n}$} & \multirow[t]{2}{*}{$\begin{array}{c}\text { Critical } \\
\text { value }\end{array}$} \\
\hline & & 60.87 & 47.73 & 71.57 & 57.33 & 69.23 & 65.80 & & & & \\
\hline Cancer Male & 60.87 & & $13.14^{*}$ & $10.7^{*}$ & 3.54 & $8.36^{*}$ & 4.93 & 6 & $\frac{4.03}{4.76}$ & 1.59 & $\frac{6.41}{7.57}$ \\
\hline $\begin{array}{l}\text { Cancer } \\
\text { Female }\end{array}$ & 47.73 & & & $23.84^{*}$ & $9.6^{*}$ & $21.5^{*}$ & $18.07^{*}$ & 5 & $\frac{3.86}{4.60}$ & 1.59 & $\frac{6.14}{7.31}$ \\
\hline $\begin{array}{l}\text { Cardiac } \\
\text { Male }\end{array}$ & 71.57 & & & & $14.24^{*}$ & 2.34 & $5.77^{* *}$ & 4 & $\frac{3.63}{4.40}$ & 1.59 & $\frac{5.77}{6.99}$ \\
\hline $\begin{array}{l}\text { Cardiac } \\
\text { Female }\end{array}$ & 57.33 & & & & & $11.9^{*}$ & $8.47^{*}$ & 3 & $\frac{3.31}{4.12}$ & 1.59 & $\frac{5.26}{6.55}$ \\
\hline $\begin{array}{l}\text { Normal } \\
\text { Male }\end{array}$ & 69.23 & & & & & & 3.43 & 2 & $\frac{2.77}{3.64}$ & 1.59 & $\frac{4.40}{5.78}$ \\
\hline $\begin{array}{l}\text { Normal } \\
\text { Female }\end{array}$ & 65.80 & & & & & & & & & & \\
\hline
\end{tabular}

Note: Mean differences were computed using Newman-Keuls formula, (Winer, 1977, PP. 191-195). $\mathrm{P}^{* *}<0.05 ; \mathrm{P}^{*}<0.01$.

- $\mathrm{r}=$ Number of Steps between ordered scores

- The critical values of q.95 and q.99 has been obtained from the distribution of Studentized Range Statistics Prepared by Leon Harter; Donald S. Clemm and Eugene H. Guthrie and reported in Winer, 1971.

The interaction effect has been graphically plotted in Figure-2 


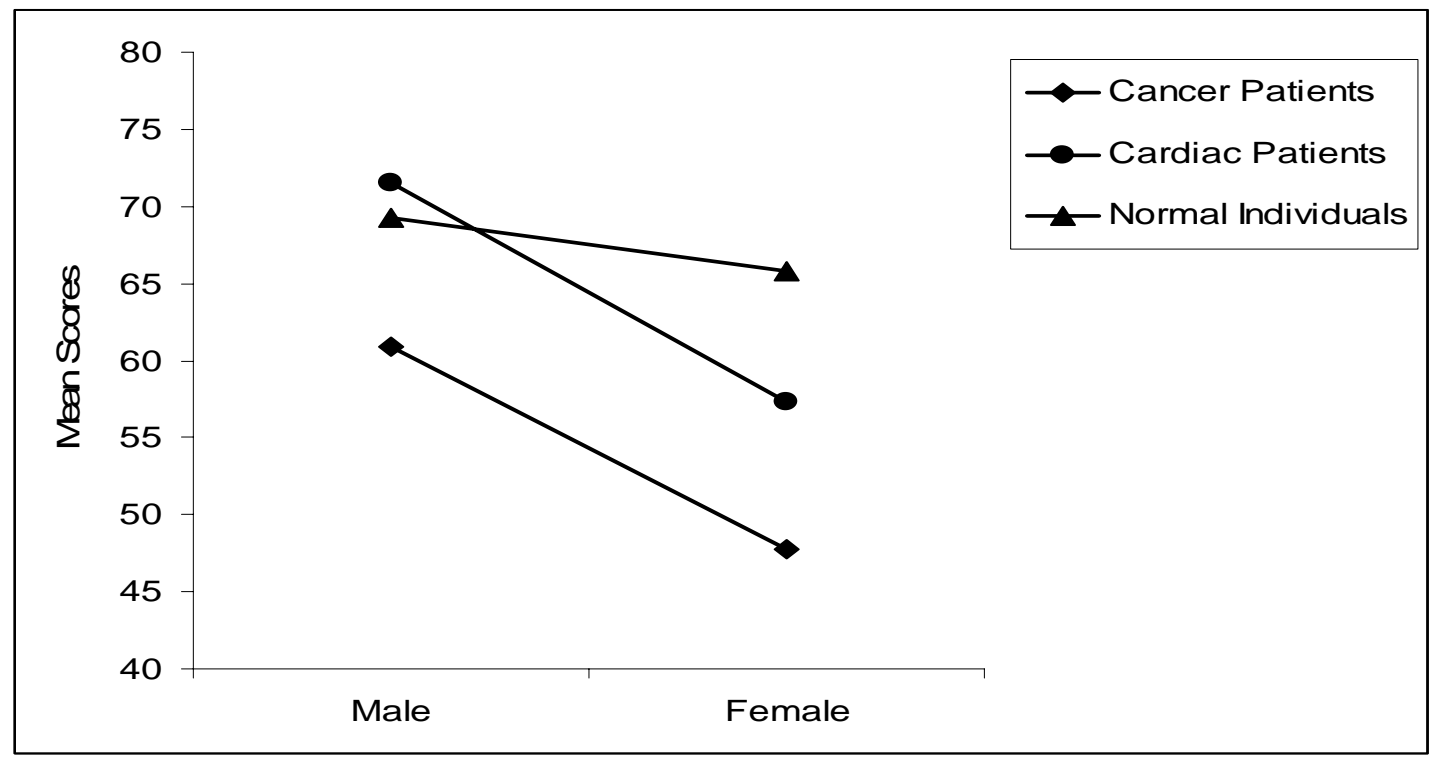

Figure 2 Showing two-way interaction effect between type of individual and gender.

Table 6 Showing overall cell means and their significant mean differences of two-way interaction involving type of individual and SES on the scores of Attitude towards Coping Scale.

\begin{tabular}{|c|c|c|c|c|c|c|c|c|c|c|c|}
\hline \multirow[t]{2}{*}{$\begin{array}{l}\text { Comparison } \\
\text { groups }\end{array}$} & \multirow[t]{2}{*}{$\begin{array}{l}\text { Mean } \\
\text { Scores }\end{array}$} & $\begin{array}{l}\text { Cancer } \\
\text { UMS }\end{array}$ & $\begin{array}{l}\text { Cance } \\
\text { r LMS }\end{array}$ & $\begin{array}{l}\text { Cardiac } \\
\text { UMS }\end{array}$ & $\begin{array}{l}\text { Cardia } \\
\text { c LMS }\end{array}$ & Normal UMS & $\begin{array}{l}\text { Normal } \\
\text { LMS }\end{array}$ & \multirow{2}{*}{$\mathbf{r}$} & \multirow[t]{2}{*}{$\frac{q .95}{q .99}$} & \multirow[t]{2}{*}{$\sqrt{m s} / n$} & \multirow[t]{2}{*}{$\begin{array}{l}\text { Critical } \\
\text { value }\end{array}$} \\
\hline & & 55.33 & 53.27 & 69.8 & 59.10 & 69.37 & 65.67 & & & & \\
\hline Cancer UMS & 55.33 & & 2.06 & $14.47^{*}$ & 3.77 & $14.04^{*}$ & $10.34^{*}$ & 6 & $\frac{4.03}{4.76}$ & 1.59 & $\frac{6.41}{7.57}$ \\
\hline Cancer LMS & 53.27 & & & $16.53^{*}$ & 5.83 & $16.1^{*}$ & $12.4^{*}$ & 5 & $\frac{3.86}{4.60}$ & 1.59 & $\frac{6.14}{7.31}$ \\
\hline Cardiac UMS & 69.8 & & & & $10.70^{*}$ & 0.43 & 4.13 & 4 & $\frac{3.63}{4.40}$ & 1.59 & $\frac{5.77}{6.99}$ \\
\hline Cardiac LMS & 59.10 & & & & & $10.27^{*}$ & $6.57^{*}$ & 3 & $\frac{3.31}{4.12}$ & 1.59 & $\frac{5.26}{6.55}$ \\
\hline Normal UMS & 69.37 & & & & & & 3.7 & 2 & $\frac{2.77}{3.64}$ & 1.59 & $\frac{4.40}{5.78}$ \\
\hline Normal LMS & 65.67 & & & & & & & & & & \\
\hline
\end{tabular}

Note: UMS=Upper Middle SES, LMS=Lower Middle SES

Note: Mean differences were computed using Newman-Keuls formula, (Winer, 1977, PP. 191-195). $\mathrm{P}^{* *}<0.05 ; \mathrm{P}^{*}<0.01$.

- $\mathrm{r}=$ Number of Steps between ordered scores

- The critical values of q.95 and q.99 has been obtained from the distribution of Studentized Range Statistics Prepared by Leon Harter; Donald S. Clemm and Eugene H. Guthrie and reported in Winer, 1971.

The interaction effect has been graphically plotted in Figure-3 


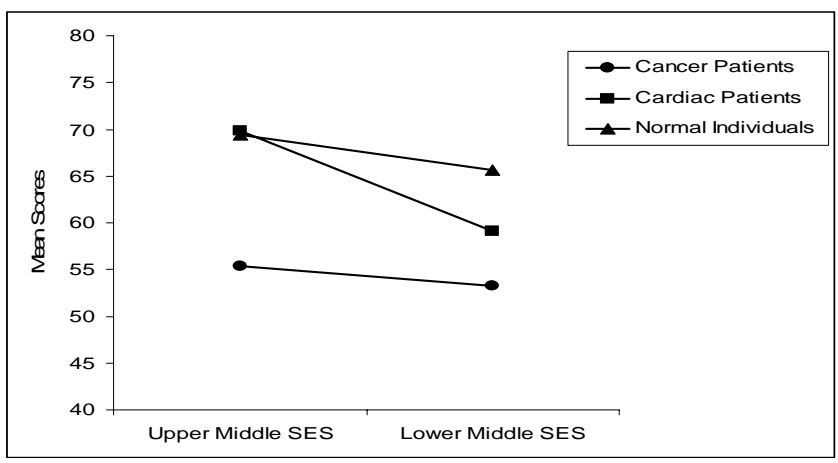

Figure 3 Showing two-way interaction effect between type of individual and SES.

\section{Discussion and Conclusion}

The present study has focused on coping attitudes of cancer and cardiac patients as a psychological phenomenon. Consequently, it has carried out an empirical investigation on different forms of coping attitudes of cancer and cardiac patients as related to socio demographic factors. The participants were male and female patients from different hospitals and health institutes of Dhaka and Rajshahi cities in Bangladesh. The first hypothesis of the present study was that both cancer and cardiac patients would express lower coping attitudes as compared to normal individuals. The findings of the present study provided empirical confirmation to this hypothesis. The results (Table 1) showed that regardless of gender and SES, cancer patients expressed significantly lower coping attitudes followed by cardiac patients and normal individuals (Table 2). These findings are in accordance with earlier studies. According to Srivastava \& Broota (1987), a good strategy to cope with cancer is to find out as much information as one can about the specific cancer. It is also a good idea to put together two support teams, the first, a support and comfort team and the other a medical and psychosocial team. A support comfort team may be family and close friends. A medicalpsychosocial team might consist of oncologists, surgeons, nurse practitioners, beside nurses, a psychologist, a psychiatrist, a social worker and clergy. A class working relationship between the medical psychosocial team members will be extremely important for purposes of coordinating treatments and for communicating and addressing an adult's needs throughout treatment (Neipp et al. 2009). Psychologists working with cancer patients and their families try to assess how well people have faired in the past and they consider not only weaknesses, but strengths. They try to work with those strengths to come up with good, compatible coping strategies, help patients develop stress management skills.
Though cardiac patients also have a tendency to show lower coping attitudes like cancer patients but the coping skills and strategies of cardiac patients are better than those of the cancer patients. Research indicates that by changing their behaviour, cardiac patients can greatly reduce their risk. When Friedman et al. (1984) showed survivors of a first heart attack how to change their behaviour, their rate of heart attacks dropped sharply compared with other survivors who had only the customary medical supervision. Over a three year period, just over 7 percent of survivors who were trained in relaxation, self-control and goal-setting had a second heart attack, compared with 13 percent of the survivors than the control group. Researchers have also examined whether patients who believe they can control their illnesses are better adjusted than those who do not see their illnesses as under personal control. Levin \& Schiller (1987) found that spirituality and religion are related to lower levels of psychological distress and reduce the risk of heart disease and also indicate lower mortality rates due to heart attacks. Sarason et al. (1994) mentioned that physical and psychological comfort provided by friends and family may play a vital role to avoid heart disease than those who remain isolated from interpersonal contact. Studies of patients with heart disease indicate that those who believe themselves able to control symptoms, health care, and treatment make a much better adjustment than do patients who believe that what happens is totally beyond their control (Thompson et al. 1993).The coping skills and strategies of normal individuals with different types of stress in their daily living activities are for better and strong than both cancer and cardiac patients. Kobasa (1989) concluded that normal individuals are able to defuse potentially stressful situations. They tend to appraise each new situation and their own ability to cope with it in positive terms. As a result, they take direct action and learn about the people and events involved. Studies have shown that progressive relaxation, biofeedback, autogenic training effectively reduces general tension \& anxiety, relieves insomnia and reduces high blood pressure (Jacob et al. 1977). 
The second hypothesis of the present study was that females would express lower coping attitudes as compared to males. The findings of the present study have provided empirical confirmation to this hypothesis. The results (Table 1) reported that regardless of type of individual and SES, females expressed significantly lower coping attitudes as compared to males (Table -3). These findings supported by earlier studies. Smith et al. (1992) reported that women use emotion focused coping, while men use problem focused coping. These differences are based on the different ways men \& women are taught to cope with the stress. Over a twelve week period Livneh et al. (1997) asked married couples to fill out a daily questionnaire dealing with events, mood, coping and so forth. The researchers found gender differences in coping and it became clear to them that women describe difficulties relevant to the self, parenting and interactions with others, while men report work-related difficulties. It was concluded that as a whole, women show lower coping attitudes than do men due to chronic illness like cancer or cardiac disorders. Wong et al. (2003) found that men tend to distract themselves using active coping strategies, whereas women use strategies involving expressing emotion. Gardner et al. (2004) found that men's response to their illness is more behavioural and dampens their depressive episodes, where women's response to their chronic illness is more ruminative and amplifies them.

The third hypothesis of the present study was that lower middle SES individuals expressed lower coping attitudes as compared to upper middle SES. The findings of the present study have provided empirical confirmation to this hypothesis. The results (Table 1) reported that regardless of type of individual and gender, lower middle SES individuals expressed lower coping attitudes as compared to upper middle SES people (Table 4). The finding may be supported from various theoretical imports as well as empirical studies. Kaur \& Murthy (1986) found a significant difference in the coping strategies among lower middle and upper middle SES people. Avoidance strategies were predominant at the life style of lower middle SES people and approach strategies were predominant at the life style of upper middle SES people. The defensive style was also used to the maximum by the lower middle SES people. Everson et al. (1998) found that lower middle SES may have indirect influence on lipid and homeostatic profiles through smoking and lack of exercise. A lack of balance between demands and buffering resource evokes negative emotions among people in lower middle SES which may in turn create bad effects on their health. Psychological stress of lower middle SES people may result in pathogenic physiological mechanisms, through nervous and endocrine processes. Carver et al. (1981) predicted that avoidant coping strategies and, possibly, emotional regulation strategies, would be more characteristic as one moves down the SES ladder, because threats from the environment may overwhelm the personal resources of the individual, or the problems, created by the environment may be largely uncontrollable; by contrast, the higher one's position on the SES ladder, the more likely one may be able to exert control over stressful events, thus leading to the deployment of active coping strategies. Bandura (1993) mentioned that upper middle SES people have a sense of self-efficacy and they also have more personal control and positive cognitions about chronic illness than people in lower middle SES. Benefits include lowering the risk of cardio-vascular disorders and lessening the negative effects of chronic diseases. A very significant feature of this study is that the average scores of attitudes towards coping of all three groups i.e. cancer, cardiac and normal individuals were below 72 obtained through the formula of computing attitudes towards coping. The reason behind this may be that the psychosocial and socio-cultural factors which are essential for maintaining proper interaction, supportive behavior, mutual understanding and trust in our familial and social life are not enough for outweighing the negative risk factors of the society. As a consequence, normal individuals apart from the chronically ill patients like cancer or cardiac disease also showed lower coping attitudes i.e. below 72 in this study though the average scores of normal individuals on attitudes towards coping were higher than that of cancer or cardiac patients.

In conclusion, this study focuses some light about the relevance of coping attitudes that appears to moderate and integrate the effects of gender and SES on developing differential attitudes towards coping of cancer and cardiac patients. Thus, it is hoped that in the absence of any specific study in the area of coping attitudes of chronically ill patients in the homogenous cultural context of Bangladesh, the investigation might provide valuable insights to the future researchers for making conclusive and valid generalizations in this important area of research.

\section{References}

Aldwin CM, Sutton KJ, Chiara G \& Spiro A. 1996. Age differences in stress, coping, and appraisal: Findings from the normative aging study. J Gerontol.Psychol Sci.51B:179-188.

Bandura A. 1993. Self-efficacy mechanism in psychobiologic functioning. Washington,DC,USA. Hemisphere Publishing Corp, 410. 
Barnoy S Bar-Tal Y and Zisser B. 2005. Correspondence in informational coping styles: How important is it for cancer patients and their spouses? Nursing Department, Tel-Aviv University, Ramat-Aviv, 69978 Tel-Aviv, Israel.

Carver CS, Pozo C \& Harris SD. 1993. How coping mediates the effect of optimism on distress: A study of women with early stage breast cancer. J Pers Soc Psychol. 65: 375-390.

Carver CS, Scheier MF, \& Weintraub J. 1981. Assessing coping strategies: A theoretically based approach. Journal of Personality and Social Psychology. 56: 267-283.

Cooper CL \& Payne R. 1978. Causes, Coping and Consequences of Stress at Work, John Wiley and Sons, New York, pp: 234-240.

Endler NS, Kocovski NL and Macrodimitris SD. 1999. Coping, efficacy, and perceived control in acute vs chronic illnesses, Journal of Chronic Diseases, Volume 52(2).

Esterling BA, Kiecolt-Glaser JK \& Glaser R. 1996. Psychosocial modulation of cytokine-induced natural killer call activity in older adults. Psychosom Med. 58: 264-272.

Everson SA, Kaplan GA, Salonen R \& Salonen JT. 1998. Does Low Socioeconomic Status Potentiate the Effects of Heightened Cardiovascular Responses to Stress on the Progression of Carotid Atherosclerosis? American Journal of Public Health. 88: 389-394.

French DP, Senior V \& Weinman J. 2001. Causal attributions for heart disease: a systematic review. Psychol Health. 16:77-98.

Friedman M, Thorensen CE \& Gill JJ. 1984. Alteration of type A behaviour and reduction in cardiac recurrences in post myocardial infraction patients. American Heart Journal: 108: 237-248.

Gardner FV \& Worwood EV. 2004. Psychological effects of cardiac surgery: a review of the literature. The Journal of the Royal Society for the Promotion of Health, 117(4): 245-249.

Gleser GC, \& Ihilevich D. 1969. An objective instrument for measuring defense mechanisms. Journal of Consulting and Clinical Psychiatry. 33: 51-60.

Goldzweig G, Hubert A, Walach N, Brenner B, Perry S, Andritsch E and Baider L. 2009. Gender and psychological distress among middle- and older-aged colorectal cancer patients and their spouses: An unexpected outcome. Critical Reviews in Oncology/Hematology, 70(1): 71-82.

Harburg E, Erfurt JC, Hauensteiri LS, Chape C, Schull WJ \& Schork MA. 1973. Socio-ecological stress, suppressed hostility, skin color, and black-white male blood pressure: Detroit. Psychosom Med. 35: 276-296.

Holland J: 1977. Psychological aspects of oncology. Med Clin N Amer. 64: 737-747.

Jacob RG, Shapiro AP \& Reeves RA. 1977. Relaxation therapy for hypertension: Comparison of effect with concomitant placebo, diuretic and B-blocker. Archives of Internal Medicine. 146: 2335-2340.

James SA \& Kleinbaum DG. 1976. Socioecologic stress and hypertension related mortality rates in North Carolina. Am. J. Public Health. 66: 354-358.

Kaur G and Murthy VN. 1986. Organizational role Stress, Coping Strategies and Locus of Control in a Major Public Sector Industrial Organization. (Unpublished Paper), Bangalore University, Bangalore.

Kobasa SC. 1989. Stressful life events, personality and health: An inquiry into hardiness. Journal of Personality and Social Psychology. 37: 1-11.

Lazarus RS \& Folkman S. 1984. Coping and adaptation. In W. D. Gentry (Ed.), Handbook of behavioral medicine (pp. 282-325). New York: Guildford Press.
Levin JS \& Schiller H. 1987. Is a Religious Attendance Really Conducive to Better Health? Toward an Epidemiology of Religion. Social Science and Medicine. 24: 589-600.

Livneh H, \& Antonak RF. 1997. Psychosocial adaptation to chronic illness and disability. Gaithersburg, MD: Aspen.

Mahler HI \& Kulik JA. 1998. Effects of preparatory videotapes on self-efficacy beliefs and recovery from coronary bypass surgery. Ann Behav Med. 20: 39-46.

Monat A, \& Lazarous RS. 1977. Introduction: Stress and coping some current issues and controversies. In A. Monat \& R. S. Lazarus (Eds.), Stress and coping: An anthology (3rd ed., pp. 115). New York: Columbia University Press.

Neipp C, Lopez-Roig S, Terol C and Pastor A. 2009. Changes in control beliefs, emotional status and psychosocial adaptation among women with breast cancer. Anales de Psycologia. 25(1): 36-43.

Parkes CM, Benjamin B \& Fitzerald RG. 1969. Broken Heart: A statistical study of increased mortality among widowers. Brit. Med. J. 1: 740-743.

Rahman A. 2010. Stress and Coping Attitudes of Cancer and Cardiac Patients as a function of Personality and Socio-demographic factors in Bangladesh, an unpublished Ph. D. Dissertation. Department of Psychology, Rajshahi University, Rajshahi, Bangladesh.

Richardson MA, Post-White J, Grimm EA, Moye LA, Singletary SE and Justice B. 1997. Coping, Life Attitudes, and Immune Responses to Imagery and Group Support after Breast Cancer Treatment, Alternative Therapies in Health and Medicine 3(5): 62-70.

Sarason IG, Levine HM, Bashan RB \& Sarason BR. 1994. Assessing Social Support: The Social Support Questionnaire. Journal of Personality and Social Psychology. 44: 127-139.

Scheier MF, Magovem GJ, Abbott RA, Matthews KA, Owens JF, Lefebvre RC, \& Carver CS. 1989. Dispositional optimism and recovery from coronary artery bypass surgery: The beneficial effects on physical and psychological well-being. Journal of Personality and Social Psychology. 57: 1024-1040.

Smith RE, Ptacek JT \& Smoll FL. 1992. Sensation Seeking, Stress and Adolescent Injuries: A Test of Stress-Buffering, RiskTaking and Coping Skills Hypotheses. Journal of Personality and Social Psychology. 62: 1016-1024.

Spiegel D. 1997. Psychosocial Aspects of Breast Cancer Treatment, Seminars in Oncology. 24: 36-47.

Srivastava S and Broota KD. 1987. Stress and Cancer; Journal of Personality and Clinical Studies. 3(2): 89-94.

Stone RA \& De Leo L. 1994. Psychotherapeutic control of hypertension. N. Engl. J. Med. 294: 80-84.

Taylor SE, Lichtman RR \& Wood JV. 1984. Attributions, beliefs about control and adjustment to breast cancer, Journal of Perspectives on Sociology and Psychology. 46: 489.

Thompson PD, Buchner D \& Pina IL. 1993. Exercise and physical activity in the prevention and treatment of atherosclerotic cardiovascular disease: a statement from the Council on Clinical Cardiology (Subcommittee on Exercise, Rehabilitation, and Prevention) and the Council on Nutrition, Physical Activity, and Metabolism (Subcommittee on Physical Activity). Circulation. 107: 3109-3116.

Williams G \& Berry JH. 1991. Chemical carcinogenesis. In: Amdur MO, Doull J, Klaassen CD, eds. Casarett and Doull's toxicology. 4th ed. New York: Pergamon Press, 127-200.

Wong WM, Cheng C, Lai KC, Wong BCY, Hu, WHC, Hui WM \& Lam SK. 2003. Psychosocial factors in patients with non cardiac chest pain. Psychosom Med. 65:443-449.

Manuscript received on 28 August 2012 and revised on 15 December 2012 